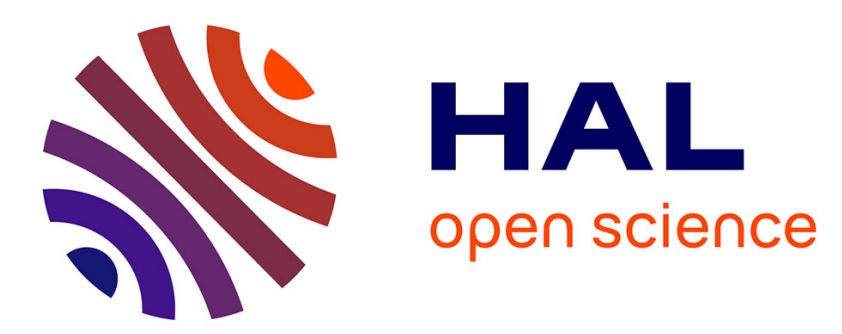

\title{
MXenes as High-Rate Electrodes for Energy Storage
}

\author{
Zifeng Lin, Hui Shao, Kui Xu, Pierre-Louis Taberna, Patrice Simon
}

\section{To cite this version:}

Zifeng Lin, Hui Shao, Kui Xu, Pierre-Louis Taberna, Patrice Simon. MXenes as High-Rate Electrodes for Energy Storage. Trends in Chemistry, 2020, 2 (7), pp.654-664. 10.1016/j.trechm.2020.04.010 . hal-03105841

\section{HAL Id: hal-03105841 \\ https://hal.science/hal-03105841}

Submitted on 11 Jan 2021

HAL is a multi-disciplinary open access archive for the deposit and dissemination of scientific research documents, whether they are published or not. The documents may come from teaching and research institutions in France or abroad, or from public or private research centers.
L'archive ouverte pluridisciplinaire HAL, est destinée au dépôt et à la diffusion de documents scientifiques de niveau recherche, publiés ou non, émanant des établissements d'enseignement et de recherche français ou étrangers, des laboratoires publics ou privés. 


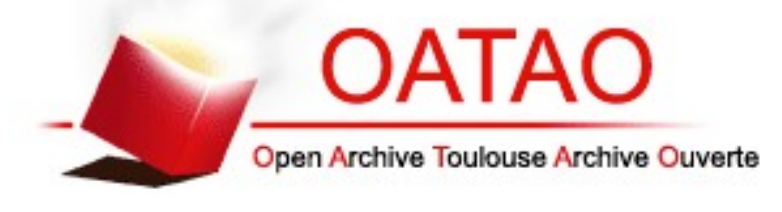

\section{Open Archive Toulouse Archive Ouverte}

OATAO is an open access repository that collects the work of Toulouse researchers and makes it freely available over the web where possible

This is an author's version published in: https://oatao.univ-toulouse.fr/27017

Official URL :

https://doi.org/10.1016/j.trechm.2020.04.010

\section{To cite this version:}

Lin, Zifeng and Shao, Hui and Xu, Kui and Taberna, Pierre-Louis and Simon, Patrice MXenes as High-Rate Electrodes for Energy Storage. (2020) Trends in Chemistry, 2 (7). 654-664. ISSN 25895974

Any correspondence concerning this service should be sent to the repository administrator: tech-oatao@listes-diff.inp-toulouse.fr 


\section{MXenes as High-Rate Electrodes for Energy Storage}

Zifeng Lin, ${ }^{1}$ Hui Shao, ${ }^{2,3}$ Kui Xu, ${ }^{4}$ Pierre-Louis Taberna, ${ }^{2,3}$ and Patrice Simon ${ }^{2,3,5, *}$

Two-dimensional transition-metal carbides/nitrides, namely MXenes, are gaining increasing interest in many research fields, including electrochemical energy storage. This short review article emphasizes some recent breakthroughs achieved in MXene chemistry and electrochemical performance when used as high-rate electrodes, especially in nonaqueous electrolytes. Lastly, the current limitations and future perspectives are highlighted.

\section{The Rise of MXenes}

MXenes are 2D materials with the formula of $\mathrm{M}_{n+1} \mathrm{X}_{n} T_{x}$, where $M$ represents the transition metal(s), $X$ is carbon and/or nitrogen, and $T_{X}$ stands for the surface terminations (e.g., $-\mathrm{OH},-\mathrm{O},-\mathrm{F}$, and so on) that are introduced during chemical preparation such as those presented in Figure $1 \mathrm{~A}, \mathrm{~B}$ [1]. Since the first discovery of the $\mathrm{Ti}_{3} \mathrm{C}_{2} \mathrm{~T}_{x}$ MXene in 2011, MXenes have expanded significantly as a novel 2D materials family containing almost 30 members with different chemical compositions and order [2]. Typically, MXenes are prepared by selective etching of the A elements from the MAX phase precursors via a HF-containing solution. Considering the rich chemistry of the MAX phase precursors as presented in Figure 1A (more than 150 MAX available today) [3-5], new MXenes are being discovered routinely. Thanks to their tunable properties (e.g., surface chemistry, conductivity, and 2D structure), MXenes are promising for many applications, including energy storage and conversion, catalysis, sensing, electromagnetics, optics, and water purification, among others [6]. However, the most promising applications deal with electrochemical energy storage, including metal-ion batteries and electrochemical capacitors (ECs) [7].

In recent years, MXenes have afforded major advances in the field of ECs, including the design of new ultra-high capacitance MXene electrodes and understanding of charge-storage mechanisms. This short review focuses on the electrochemical behavior of MXene electrodes in aqueous and nonaqueous electrolytes for energy-storage applications. The state-of-the-art findings of the intercalation pseudocapacitance of the MXene electrode in lithium- or sodium-containing organic electrolytes will be also described, which paves the way for preparing high energy-density MXene-based ECs for practical applications.

\section{Advancements in MXene Chemistry}

Different from the well-known 2D graphene or other 2D materials that are stacked and held together by van der Waals interactions and are easily exfoliated from their corresponding precursors, the chemical bonds between the $\mathrm{M}_{n+1} \mathrm{X}_{n}$ layer and A element layer of MAX phases are much stronger, resulting in difficulties for preparing MXenes via mechanical exfoliation. However, the bonding of $\mathrm{M}-\mathrm{X}$ atoms is much stronger than that of the $\mathrm{M}-\mathrm{A}$ atoms [8], enabling the possibility of selective removal of the A element with specific chemical etchants. In 2011, Gogotsi, Barsoum, and colleagues first reported the synthesis of $\mathrm{Ti}_{3} \mathrm{C}_{2} \mathrm{~T}_{x}$ (the first MXene), prepared by selective etching of Al-layers from the $\mathrm{Ti}_{3} \mathrm{AlC}_{2} \mathrm{MAX}$ phase precursor immersed in HF solution [9]. Later, alternative acidic solutions were proposed as etching baths, such as the mixture of hydrochloric acid $(\mathrm{HCl})$ with lithium fluoride (LiF) [10], $\mathrm{HCl}$ with sodium fluoride (NaF) [11], ammonium hydrogen

\section{Highlights}

MXenes are $2 \mathrm{D}$ materials that offer great promise for electrochemical energy storage. While MXene electrodes achieve high specific capacitance and power rate performance in aqueous electrolytes, the narrow potential window limits the practical interest of these systems.

The development of new synthesis methods to prepare MXenes, such as by using a molten salt approach, broadens the variety of precursors that can be used. In addition, these methods allow for tuning the nature and content of the surface functional groups present at the MXene surface.

Recent studies reported high performance for MXene electrodes in nonaqueous electrolytes with both high capacity, high voltage, and fast chargedischarge rate. Those performances, ascribed to the control of the MXene/ electrolyte interface, offer new opportunities for designing the next generation of high-rate materials for energy storage applications.

${ }^{1}$ College of Materials Science and Engineering, Sichuan University, No. 24 South Section 1, Yihuan Road, Chengdu 610065, PR China

${ }^{2}$ CIRIMAT, Université de Toulouse, CNRS, CIRIMAT, 118, Route de Narbonne, 31062 Toulouse Cedex, 9, France

${ }^{3}$ Réseau sur le Stockage Electrochimique de l'Energie (RS2E), Fédération de Recherche CNRS 3459, HUB de l'Energie, Rue Baudelocque, 80039 Amiens, France ${ }^{4}$ Key Laboratory of Flexible Electronics (KLOFE) and Institute of Advanced Materials (IAM), Jiangsu National Synergetic Innovation Center for Advanced Materials (SICAM), Nanjing Tech University (NanjingTech), 30 South Puzhu Road, Nanjing 211816, PR China

Institut Universitaire de France, 1, Rue des Ecoles, 75005 Paris, France

${ }^{*}$ Correspondence: simon@chimie.ups-tlse.fr (P. Simon). 
(A)

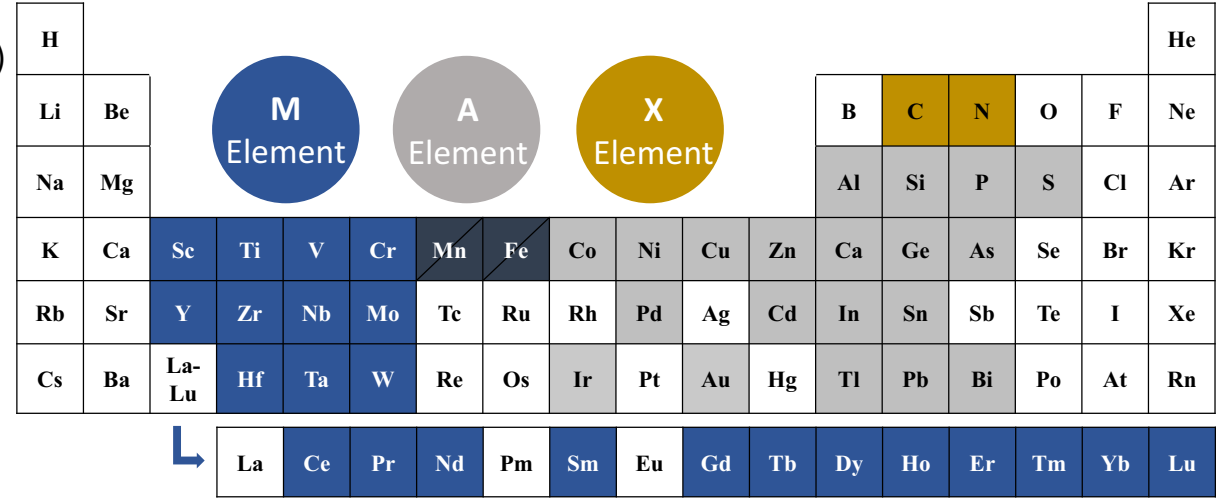

(B)

$\mathrm{Ti}_{3} \mathrm{AIC}_{2} \mathrm{MAX}$

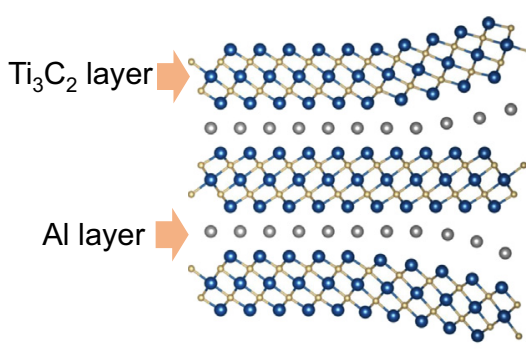

$\mathrm{Ti}_{3} \mathrm{C}_{2} \mathrm{~T}_{x} \mathrm{MXene}$

$\therefore: \dot{a}: \dot{a}: \dot{a}:$ \%8: :․․․?

$\dot{0} \dot{0} \dot{0} \dot{0} \dot{0} \dot{0} \dot{0}$

008008080

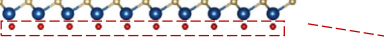

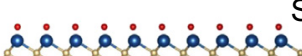
Surface groups 6. $6060 \%$

Trends in Chemistry

Figure 1. The MAX Phase and MXene Chemistry. (A) Periodic table of the elements showing the updated MAX phase compositions, including the recently reported novel MAX phases with $\mathrm{A}=\mathrm{Co}, \mathrm{Ni}, \mathrm{Cu}, \mathrm{Zn}$, and so on; Mn and Fe may appear in either $\mathrm{M}$ or $\mathrm{A}$ position [4,5]. (B) Schematic showing the etching of $\mathrm{Ti}_{3} \mathrm{AlC}_{2} \mathrm{MAX}$ phase precursor into $\mathrm{Ti}_{3} \mathrm{C}_{2} \mathrm{~T}_{X} \mathrm{MXene}$.

fluoride $\left(\mathrm{NH}_{4} \mathrm{HF}_{2}\right)$ [12], and ammonium dihydrogen fluoride in organic polar solvents [13]. To avoid the use of hazardous HF-containing solutions, other methods such as hydrothermal treatment in aqueous sodium hydroxide ( $\mathrm{NaOH}$ ) solution [14] or thermal-assisted electrochemical etching [15] were developed. While these methods can be successfully used for Al-containing MAX phases (Al as the A element), as presented in Figure $1 \mathrm{~A}$, other MAX phases (with $\mathrm{A}=\mathrm{Si}, \mathrm{Ga}, \mathrm{Ge}, \mathrm{Sn}$, and so on) are still difficult to etch. Some progress has been made, such as the successful etching of the $\mathrm{Ti}_{3} \mathrm{SiC}_{2} \mathrm{MAX}$ phase by a HF solution containing $\mathrm{H}_{2} \mathrm{O}_{2}$ [16]. Recently, $\mathrm{Li}$ and colleagues [17] reported a method to prepare $2 \mathrm{D}$ MXenes from a broad range of MAX-phase precursors, with $\mathrm{A}$ elements $\mathrm{Al}, \mathrm{Si}, \mathrm{Ga}$, and $\mathrm{Zn}$, based on the MAX precursors etching in F-free Lewis acid molten salts (see Glossary). Interestingly, theoretical calculations show the possibility of preparing MXenes from unexplored MAX phases by a careful selection of the Lewis acid salts, which exhibit a redox potential higher than that of the corresponding A elements. This method sheds light on the opportunity to further enrich the MXene family.

Also important to note is that a change in the synthesis route of MXenes may result in a noticeable difference in surface terminations [18]. For instance, the surface terminations of $\mathrm{HF}$ - or HCl/LiFetched MXenes mainly contain -O, -OH, and -F. Quantitative NMR spectroscopy has shown that HF-synthesized $\mathrm{Ti}_{3} \mathrm{C}_{2} \mathrm{~T}_{x}$ MXene exhibit almost four times $-\mathrm{F}$ termination as well as more -OH and less - $\mathrm{O}$ terminations compared with the same $\mathrm{Ti}_{3} \mathrm{C}_{2} \mathrm{~T}_{x}$ MXene prepared in a mixture of $\mathrm{HCl} / \mathrm{LiF}$ solution [19]. Even using the same method with different $\mathrm{HF}$ concentrations may result in the variation in surface termination composition [20]. MXenes prepared by $\mathrm{NaOH}$ hydrothermal treatment [14] or electrochemical etching [15] show no -F termination on the surface, and Lewis 
acid molten salts-derived MXenes present mainly - $\mathrm{Cl}$ and -O surface terminations [17]. Annealing treatment of MXenes under vacuum or various gas atmospheres is an effective route to manipulate surface terminations [21-24]. Persson and colleagues reported the possibility of defluorination by heating under vacuum conditions and further fully terminated with oxygen by exposure to oxygen gas at $450^{\circ} \mathrm{C}$ [24]. Both theoretical and experimental investigations show that the change in surface chemistry has important effects on the resulting physical or chemical properties (e.g., conductivity and chemical redox activity) [21,23,25]. For instance, the hydrophilicity of MXenes depends greatly on the surface terminations: MXenes prepared by HF or HCl/LiF are typically hydrophilic due to the existence of $-\mathrm{OH}$ or $-\mathrm{O}$ functional groups $[1,10]$, which can be turned hydrophobic by further removal of these groups upon thermal treatment [26].

Most MXenes exhibit a metal-like conductivity; some semimetallic or semiconducting MXenes have also been identified, depending on the nature of $\mathrm{M}$ elements and the surface terminations [25]. $\mathrm{Ti}_{3} \mathrm{C}_{2} \mathrm{~T}_{x}$ spin-cast MXene films exhibit a high conductivity of about $10^{4} \mathrm{~S} \mathrm{~cm}^{-1}$ [27]. Another key feature making MXenes attractive candidates for EC electrodes is their unique 2D structure with open accordion-like morphology and high specific surface area, which allows for fast charge transport and high surface charge accumulation.

\section{MXenes as EC Electrodes in Aqueous Electrolytes}

Unsurprisingly, the first discovered MXene, $\mathrm{Ti}_{3} \mathrm{C}_{2} \mathrm{~T}_{x}$, is to-date the most studied. In 2013, Gogotsi and colleagues reported successful accommodation of a large variety of cations, including $\mathrm{Li}^{+}, \mathrm{Na}^{+}$, $\mathrm{K}^{+}, \mathrm{NH}_{4}^{+}, \mathrm{Mg}^{2+}$, and $\mathrm{Al}^{3+}$, in a $\mathrm{Ti}_{3} \mathrm{C}_{2} \mathrm{~T}_{x} \mathrm{MXene}$ electrode, from neutral and alkaline aqueous electrolytes. A volumetric capacitance exceeding $300 \mathrm{~F} \mathrm{~cm}^{-3}$ was achieved, corresponding to about three times that of conventional porous carbons [28]. The symmetric and rectangular-shaped voltammograms observed in various alkaline and neutral electrolytes (Figure 2A and Box 1), without marked redox peaks, indicate a typical capacitive electrochemical signature. Moving to acidic aqueous electrolytes, Ghidiu and colleagues reported capacitances up to $900 \mathrm{~F} \mathrm{~cm}^{-3}\left(245 \mathrm{~F} \mathrm{~g}^{-1}\right)$ in $1 \mathrm{M}$ $\mathrm{H}_{2} \mathrm{SO}_{4}$ electrolyte without significant degradation over 10000 cycles [10]. An exceptional high capacitance of $1500 \mathrm{~F} \mathrm{~cm}^{-3}\left(370 \mathrm{~F} \mathrm{~g}^{-1}\right)$ was further achieved within a 1-V potential range, outperforming the best reported pseudocapacitive or capacitive electrode materials in aqueous electrolytes [29]. As presented in Figure 2B, a pair of redox peaks were observed on the cyclic voltammetry (CV) profiles, indicating the pseudocapacitive nature of the $\mathrm{Ti}_{3} \mathrm{C}_{2} \mathrm{~T}_{x}$ MXene electrode in acidic aqueous electrolytes. In situ X-ray absorption spectroscopy measurements further evidenced the variation of the Ti oxidation state of the $\mathrm{Ti}_{3} \mathrm{C}_{2} \mathrm{~T}_{x} \mathrm{MXene}$ electrode during electrochemical polarization, confirming the pseudocapacitive charge-storage mechanism [30]. Further studies by $\mathrm{Hu}$ and colleagues revealed that hydronium in the $\mathrm{H}_{2} \mathrm{SO}_{4}$ electrolyte (de)bonds with the terminal - $\mathrm{O}$ in the $\mathrm{Ti}_{3} \mathrm{C}_{2} \mathrm{~T}_{x} \mathrm{MXene}$ electrode upon discharging(charging) that gives rise to the change of oxidation state of titanium [31]. In addition to the high capacitance and enlarged potential window, the extremely high-rate performance was achieved by controlling the electrode porous structure to enhance electrolyte accessibility to the MXene surface. The macroporous $\mathrm{Ti}_{3} \mathrm{C}_{2} \mathrm{~T}_{x} \mathrm{MXene}$ electrode (Figure 2C) can still deliver a capacitance of $210 \mathrm{~F} \mathrm{~g}^{-1}$ at $10 \mathrm{~V} \mathrm{~s}^{-1}$ [29]. Designing vertically aligned $\mathrm{Ti}_{3} \mathrm{C}_{2} \mathrm{~T}_{x} \mathrm{MXene}$ flakes onto the current collectors could further boost the rate performance, as shown in Figure 2D. The electrode architecture greatly shortens the ion transport pathway as compared with a conventional filtered film electrode. As a result, CV profiles in Figure 2E clearly evidence the excellent performance of a vertically aligned $\mathrm{Ti}_{3} \mathrm{C}_{2} \mathrm{~T}_{x} \mathrm{MXene}$ electrode, even with an electrode thickness up to $200 \mu \mathrm{m}$ [32]. In addition to $\mathrm{Ti}_{3} \mathrm{C}_{2} \mathrm{~T}_{X} \mathrm{MXene}$, other MXenes such as $\mathrm{Ti}_{2} \mathrm{C}, \mathrm{Mo}_{2} \mathrm{C}$, $\mathrm{Mo}_{1.33} \mathrm{C}, \mathrm{V}_{2} \mathrm{C}$, and so on, were also investigated in aqueous electrolytes and fascinating electrochemical performance was reported [33-37]. For example, VahidMohammadi and colleagues showed that $\mathrm{V}_{2} \mathrm{CT}_{x} \mathrm{MXene}$ electrodes are able to deliver high gravimetric $\left(\sim 400 \mathrm{~F} \mathrm{~g}^{-1}\right)$ and volumetric $\left(\sim 1300 \mathrm{~F} \mathrm{~cm}^{-3}\right)$ capacitance in $\mathrm{H}_{2} \mathrm{SO}_{4}$ electrolytes, even surpassing the performance of $\mathrm{Ti}_{3} \mathrm{C}_{2} \mathrm{~T}_{x}$

\section{Glossary}

Cyclic voltammetry (CV): a widely used electrochemical technique. The principle is to apply a linear voltage ramp to an electrode (or a device) between two voltage limits and measure the resulting current.

Energy density: the amount of energy stored in a given energy storage device normalized with the mass or volume. The Sl unit is $\mathrm{J} / \mathrm{m}^{3}$ or $\mathrm{J} / \mathrm{kg}(\mathrm{Wh} / \mathrm{l}, \mathrm{Wh} / \mathrm{kg}$ are commonly used for batteries and supercapacitors).

Galvanostatic charge-discharge (GCD): an electrochemical technique of applying a constant to charge/discharge the electrochemical cell or electrode within the control potential range. Ionic liquids: salts composed solely of ions with melting points below $100^{\circ} \mathrm{C}$ LP30: 1.0 M lithium

hexafluorophosphate $(\mathrm{Li}-\mathrm{PF} 6)$ in ethylene carbonate $(\mathrm{EC})$ and dimethyl carbonate (DMC) organic solvents with a volume ratio of 1:1.

Molten salts: salts composed solely of ions, but with higher melting points. Specific capacitance: capacitance is the ratio of the charge change of a system to the corresponding potential change. In supercapacitors, capacitances of the electrode (or cell) are typically normalized with the electrode (or cell) mass, volume, and areal, which are termed as specific gravimetric, areal, and volumetric capacitance, respectively. 


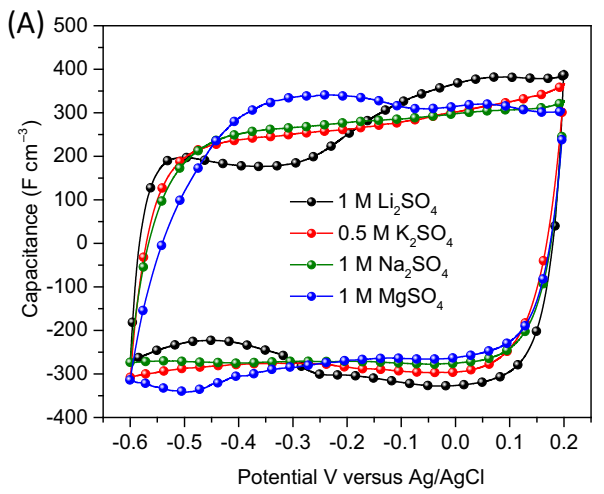

(D)

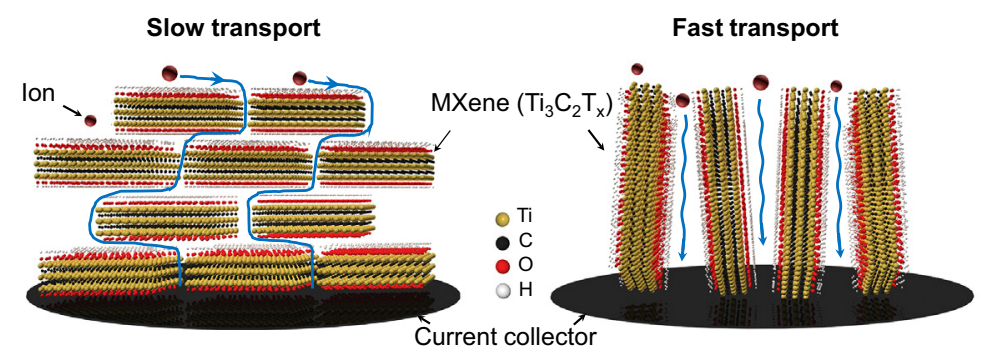

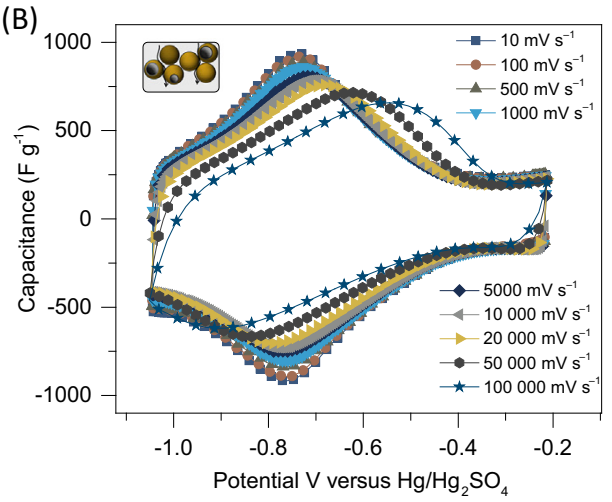

(C)
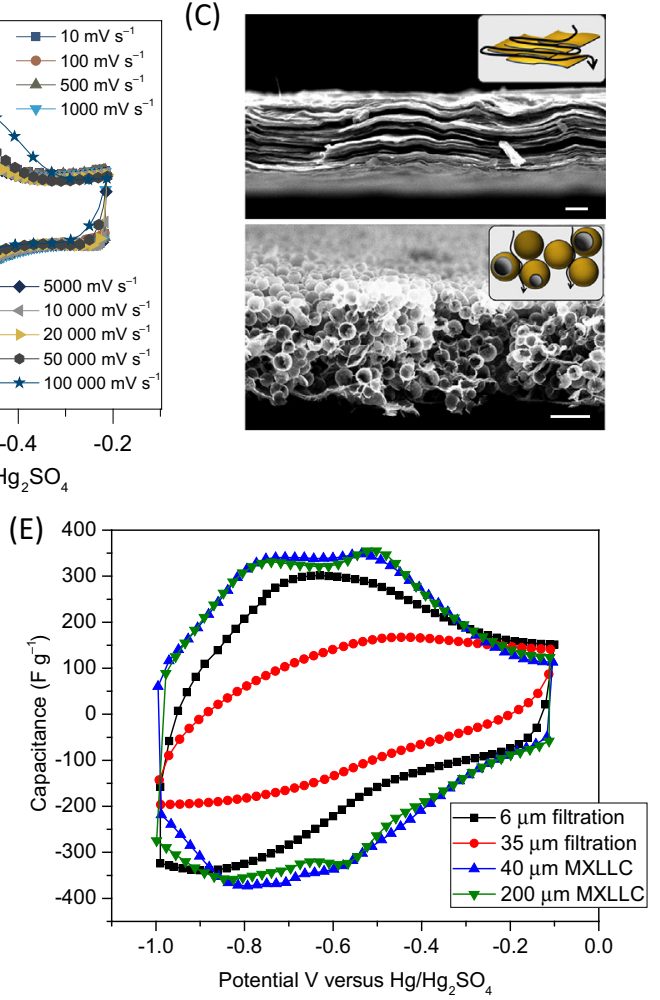

Trends in Chemistry

Figure 2. Electrochemical Signature of MXenes in Aqueous Electrolytes. (A) Cyclic voltammograms at $2 \mathrm{mV} \mathrm{s}^{-1}$ for a $25-\mu m$-thick $d-T_{3} \mathrm{C}_{2} \mathrm{~T}_{x}$ paper electrode in sulfate aqueous electrolytes. (B) Cyclic voltammetry data collected at scan rates from 10 to $100000 \mathrm{mV} \mathrm{s}{ }^{-1}$ for a 90-nm-thick $\mathrm{Ti}_{3} \mathrm{C}_{2} \mathrm{~T}_{x} \mathrm{MXene}$. (C) Scanning electron microscopy images of a (top panel) $\mathrm{Ti}_{3} \mathrm{C}_{2} \mathrm{~T}_{x}$ MXene hydrogel cross-section and (bottom panel) macroporous templated $\mathrm{Ti}_{3} \mathrm{C}_{2} \mathrm{~T}_{x}$ electrode cross-section. Scale bars: 5 $\mu \mathrm{m}$. Insets show a schematic representation of the ionic current pathway in electrodes of different architectures. (D) Schematic illustration of ion transport in horizontally stacked and vertically aligned $\mathrm{Ti}_{3} \mathrm{C}_{2} \mathrm{~T}_{x}$ MXene electrodes. (E) Cyclic voltammograms of the indicated samples at a scan rate of $100 \mathrm{mV} \mathrm{s}^{-1}$. Adapted, with permission, from $[1,28,29,32]$.

electrodes in similar electrolytes [33]. These results highlight the importance of developing a larger portfolio of MXenes with appealing properties and shed light on the promising future of MXenes for capacitive energy storage.

However, a limitation comes from the narrow potential window, limited on one hand to the hydrogen evolution reaction in the negative potential range and on the other hand to MXene oxidation at higher anodic potentials. As a result, despite high specific capacitance performance, the maximum energy density $E\left(E=1 / 2 C \cdot V^{2}\right.$, where $C$ is the capacitance and $V$ is the maximum operating voltage) still falls short.

\section{MXenes as High-Rate Electrodes in Nonaqueous Electrolytes}

A direct approach to broaden the cell voltage is to move from aqueous to nonaqueous electroIytes. Generally, nonaqueous electrolytes, including organic and ionic liquid electrolytes that are stable within a potential window up to $3 \mathrm{~V}$ (or higher). Conventional organic electrolytes for ECs consist of acetonitrile (ACN) or propylene carbonate (PC) as solvents and conducting salts

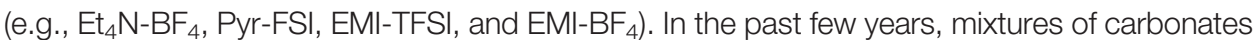
in combination with lithium or sodium salts that are typically used as electrolytes in lithium- or sodium-ion batteries have been proposed as electrolytes for ECs as well. 
Box 1. Batteries and Electrochemical Capacitors (ECs)

ECs include electrical double-layer capacitors (EDLCs) and pseudocapacitors. EDLCs store charge through electrostatic adsorption/desorption of charge carriers on porous electrodes and electrolyte interfaces, while pseudocapacitors store charge mainly through redox reactions of electrode materials with electrolytes. Batteries use diffusion-limited electrochemical oxidation-reduction (redox) reactions to store the charge, where phase transition in the materials is usually achieved during charge-discharge. Current batteries include lithium-ion, sodium-ion, and zinc-ion batteries (under development), while potassium-ion, aluminum-ion, and magnesium-ion batteries are currently at the research stage. As recently discussed by Mathis and colleagues [58], capacitive/pseudocapacitive systems can be distinguished from battery-like materials by comparing their electrochemical signatures, such as cyclic voltammograms (CVs) and galvanostatic charge-discharge (GCD). EDLC electrode materials typically show a linear voltage versus time response (a triangularshaped profile) during constant current charging/discharging (Figure IA) and rectangular CVs (Figure ID). A constant capacitance is achieved in the full potential range. However, battery-type electrode materials show obvious plateaus during GCD profiles (Figure IC) or sharp, clearly separated oxidation and reduction peaks (Figure IF) in CV curves. In such cases, the use of capacitance $(F)$ is meaningless since a capacitance defines a constant change of the charge $Q(A s)$ in a potential window $V$ (Figure IA). The capacity $Q$ (A.S) has then to be employed for this general case. Pseudocapacitance, also called 'redox capacitance', is a charge storage mechanism involving fast, nondiffusion limited redox reactions. When the storage is limited to the surface, pseudocapacitive materials show box-like $\mathrm{CV}$ response $\left(\mathrm{RuO}_{2}, \mathrm{MnO}_{2}\right)$; the capacitance $(F)$ can then be used to define the electrochemical behavior. When these fast, nondiffusion limited redox reactions occur within the bulk of the material, they are termed intercalation pseudocapacitance. Materials like MXenes [29], $\mathrm{Nb}_{2} \mathrm{O}_{5}$ [45], and $\mathrm{WO}_{3}$ [59], show a sloping discharge profile (Figure IB) and CVs with the presence of broad, symmetric redox peaks (Figure IE). In these cases, the use of capacity $Q$ (As or mAh) is recommended to account for the redox nature of the charge storage. There are also many methods that have been proposed to categorize the electrode by examination of the material's intrinsic charge storage kinetics [58,60-62].
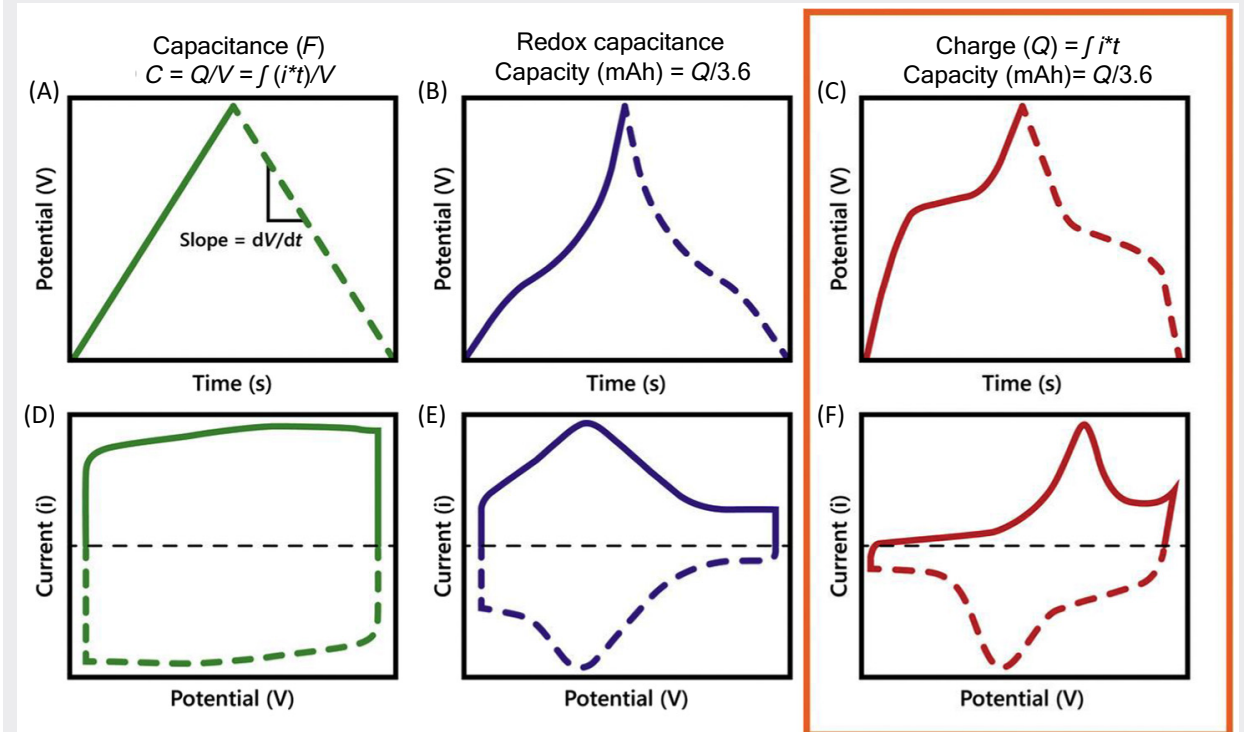

Trends in Chemistry

Figure I. Archetypal Electrical Output Behavior of Three Main Types of Electrodes, Including (A,D) Electrical Double Layer, (B,E) Pseudocapacitive, and (C,F) Battery Type. (A-C) Schematic of galvanostatic chargedischarge profiles showing linear and nonlinear responses with time and (D-F) corresponding cyclic voltammetry profiles. Adapted, with permission, from [58].

In Conventional Nonaqueous Electrolytes

Early efforts devoted to the investigation of MXenes in conventional nonaqueous electrolytes achieved a significant extension of the potential window [38-40]. In 2015, Dall'Agnese and colleagues tested MXene/carbon nanotube (MXene/CNT) composite electrodes in several organic electrolytes, including $1 \mathrm{M} \mathrm{EMI-TFSI,} 1 \mathrm{M} \mathrm{EMI-BF}_{4}$, and $1 \mathrm{M} \mathrm{TEA-BF}_{4}$ in ACN [40]. A potential win-dow of $1.8 \mathrm{~V}$ was achieved, together with a limited maximum capacitance of $80 \mathrm{~F} \mathrm{~g}^{-1}(245 \mathrm{~F}$ $\mathrm{cm}^{-3}$ ). 
CNTs were used here to avoid the restacking of the MXene electrode film obtained by vacuum filtration and enhance the accessibility of the MXene surface to electrolyte ions, including those of large size. Interestingly, a pair of redox peaks were observed, which could be ascribed to the intercalation of cations. Later on, Lin and colleagues [39] prepared a $\mathrm{Ti}_{3} \mathrm{C}_{2} \mathrm{~T}_{x} \mathrm{MXene}$ electrode preintercalated with a neat ionic liquid electrolyte (EMI-TFSI) that could deliver high power capability within a $3 \mathrm{~V}$ voltage window with a capacitive electrochemical signature; however, the capacitance was still limited and reached only $80 \mathrm{~F} \mathrm{~g}^{-1}$. Moreover, similar redox peaks were also observed in the CV profiles of the electrode when tested in a three-electrode cell set-up, as presented in Figure $3 \mathrm{~A}$. Further in situ X-ray diffraction (XRD) analysis indicates the reversible expansion of interlayer spacing during negative polarization, while a reversible shrinkage occurs during positive polarization. Such behavior was explained by the reversible (de)intercalation of $E M I^{+}$cations at the $\mathrm{Ti}_{3} \mathrm{C}_{2} \mathrm{~T}_{x}$ negative electrode and $\mathrm{EMI}^{+}$cation/TFSI ${ }^{-}$anion exchange at $\mathrm{Ti}_{3} \mathrm{C}_{2} \mathrm{~T}_{x}$ positive electrode (Figure 3B) $[41,42]$. This charge-storage mechanism was later confirmed by molecular dynamic simulations [43]. In addition, by combining electrochemical dilatometry together with in situ XRD measurements, Jäckel and colleagues demonstrated a highly reversible swelling/contraction of $\mathrm{Ti}_{3} \mathrm{C}_{2}{ }^{-}$ MXenes in two different neat ionic liquids (EMI-TFSI and BMIM-BF 4 ). Figure 3C,D presents the

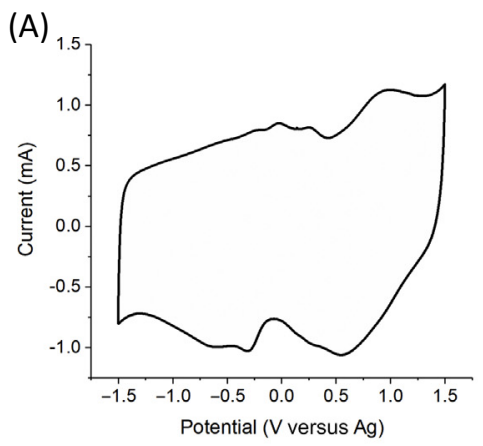

(C)

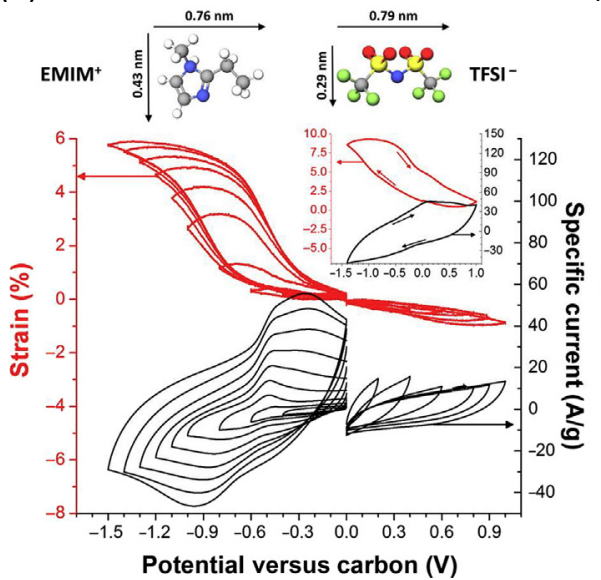

(B)

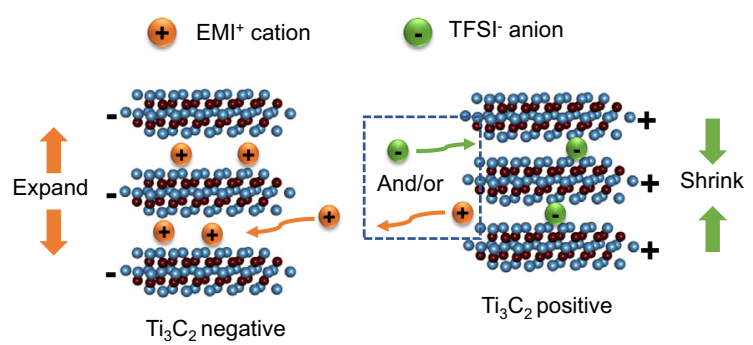

(D)

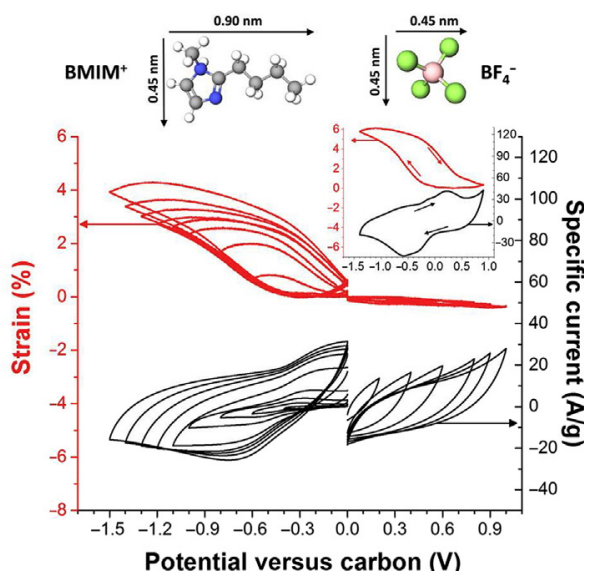

Trends in Chemistry

Figure 3. MXene Electrochemical Capacitor (EC) Electrodes in Conventional Nonaqueous Electrolytes. (A,B) $\mathrm{Ti}_{3} \mathrm{C}_{2} \mathrm{~T}_{x}$ electrodes in neat EMI-TFSI ionic liquid electrolyte. (A) Cyclic voltammetry (CV) profile of at $20 \mathrm{mV} \mathrm{s}^{-1}$ in the -1.5 to $1.5 \mathrm{~V}$ versus $\mathrm{Ag}$ potential range. (B) Schematic of the charge storage mechanism-cation insertion at negative polarization and cation desertion and/or anion insertion at positive polarization. (C,D) Combination of in situ electrical dilatometry and cyclic voltammetry at $1 \mathrm{mV} \mathrm{s}^{-1}$ sweep rate. The expansion/contraction is normalized to the equilibrium thickness (after $24 \mathrm{~h}$ ) at $0 \mathrm{~V}$

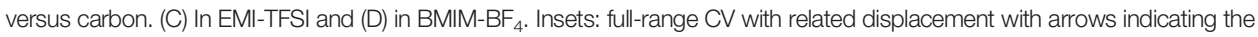
direction of potential sweeping. Adapted, with permission, from $[41,42,44]$. 
strain evolution of the MXene electrode during CV tests in two ionic liquids that confirm much larger stain during cation insertion under negative polarization, in comparison with cation/anion swapping during positive polarization [44]. Recently, Zheng and colleagues used ionic liquid preintercalated MXene films as electrodes to prepare ionogel-based flexible micro-supercapacitors (MSCs) [38]. MSCs could achieve a cell voltage of $3 \mathrm{~V}$, together with volumetric and areal energy density among the highest values reported for MSCs (43.7 $\mathrm{mWh} \mathrm{cm}^{-3}$ and $13.9 \mu \mathrm{Wh} \mathrm{cm}{ }^{-2}$, respectively).

To summarize, limited attention has been focused on MXene-based ECs in conventional organic or ionic liquid electrolytes, which have the potential to change the game because of the large voltage windows associated with nonaqueous electrolytes. Although larger potential windows up to $3 \mathrm{~V}$ can be obtained, the specific capacitance is still limited as compared with those achieved in aqueous electrolytes. A possible explanation is the restacking of the MXene flakes, resulting in narrower interlayer spacing and consecutive limited cation/anion access to the interlayer space between the MXene sheets. Fine-tuning the interlayer spacing or designing a specific electrode structure adapted to the electrolyte may appear as effective routes to enhance the performance in these electrolytes. Another promising strategy is to unlock the redox activity of MXenes in nonaqueous electrolytes as observed in aqueous acidic electrolytes.

In Metal lon-Containing Organic Electrolytes

As reported by Dunn and colleagues, some metal oxides, such as $\alpha-\mathrm{MoO}_{3}$ and $\mathrm{T}-\mathrm{Nb}_{2} \mathrm{O}_{5}$, can act as a lithium storage host via ultrafast lithium-ion (de)intercalation in Li-ion battery electrolytes, without diffusion limitations $[45,46]$. Those materials offer great promise to enhance the rate capability (in charge and discharge) of Li-ion batteries. Considering their accordion-like open structure and metallic conductivity, MXenes were expected to be good candidates for fast Li-ion intercalation. As a result, since the first publication of MXenes by Naguib and colleagues from M. Barsoum's and Y. Gogotsi's groups, they have been studied as a negative electrode for Liion battery applications [47-50]. Figure 4A shows a typical $\mathrm{CV}$ of $\mathrm{a} \mathrm{Ti}_{3} \mathrm{C}_{2} \mathrm{MXene}$ electrode prepared from etching in HF- or F-containing solution (noted as HF-MXene) [50]. The lithium intercalation/deintercalation reaction (Figure 4A) occurs within the full potential window, that is, from 0.05 $\mathrm{V}$ up to $3 \mathrm{~V}$ versus $\mathrm{Li}^{+} / \mathrm{Li}$, and is achieved in a sequential way through several steps [50]. The CV shows two different sets of peaks visible at 2.53/2.45 $\mathrm{V}$ versus $\mathrm{Li}^{+} / \mathrm{Li}$ and 1.00/0.97 $\mathrm{V}$ versus $\mathrm{Li}^{+}$/ $\mathrm{Li}$. According to the previous study [50], where combined electrochemical tests and in situ XRD study were achieved, the set of redox peaks at high potential corresponds to Li-ion intercalation in large interlayer space, while the peaks at low potential correspond to Li-ion intercalation in small interlayer space. However, the large operating potential window for a negative electrode, together with the high irreversible capacity at the first cycle, has so far drastically limited the interest of these HF-MXene materials in Li-ion battery applications.

MXenes have also been studied as a positive electrode for sodium-ion intercalation. Figure 4B shows the $\mathrm{CV}$ of a $\mathrm{V}_{2} \mathrm{C} M$ Xene electrode in $1 \mathrm{M} \mathrm{Na}^{-P F_{6}}$ in EC/DMC [51]. A set of redox peaks associated with $\mathrm{Na}^{+}$intercalation/deintercalation reaction are visible at about $2.5 \mathrm{~V}$ versus $\mathrm{Na}^{+}$/ $\mathrm{Na}$, on top of a rectangle-shaped capacitive signature from 1 to $3 \mathrm{~V}$ versus $\mathrm{Na}^{+} / \mathrm{Na}$. A similar electrochemical process was also observed with the $\mathrm{Ti}_{2} \mathrm{C} M$ Xene electrode in $1 \mathrm{M} \mathrm{Na}-\mathrm{PF}_{6}$ in EC/DEC, where a reversible capacity of $175 \mathrm{mAh} \mathrm{g}^{-1}$ was delivered in the 0.1-2.3V potential range [52]. Kajiyama and colleagues found that the intercalation distance in MXene electrode is expanded by the first sodiation process because of desolvated $\mathrm{Na}^{+}$intercalation and solvent molecule penetration, which further act as pillars to stabilize the structure [53]. Porous MXene electrodes were also developed to accelerate ion transport in organic electrolytes [54-56]. To summarize, similar to Li-ion, Na-ion intercalation in MXene electrodes is mainly achieved within a large operating potential window, which is a concern for further use in Na-ion battery applications. 

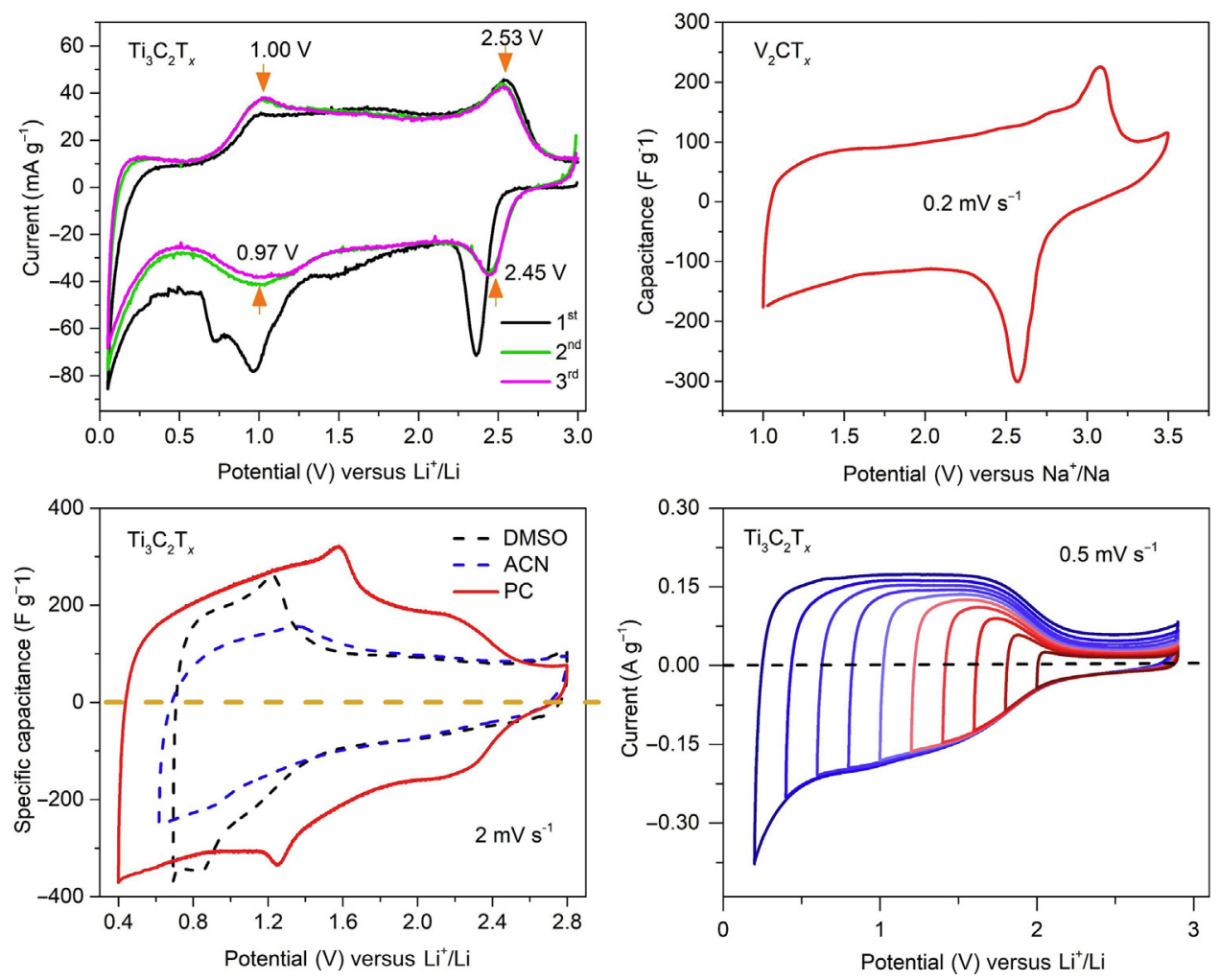

Figure 4. MXene Electrodes in Lithium or Sodium-Containing Organic Electrolytes. Cyclic voltammetry profiles of (A) $\mathrm{Ti}_{3} \mathrm{C}_{2} \mathrm{~T}_{x}$ in LP30; (B) $\mathrm{V}_{2} \mathrm{CT}_{x}$ in $1 \mathrm{M} \mathrm{NaPF}_{6}$ in EC/DMC (1:1); (C) $\mathrm{Ti}_{3} \mathrm{C}_{2} \mathrm{~T}_{x}$ in $1 \mathrm{M} \mathrm{Li-TFSI}$ in DMSO, ACN, PC solvents, the original potential versus Ag wire is converted into potential versus $\mathrm{Li}^{+} / \mathrm{Li}$; (D) $\mathrm{Ti}_{3} \mathrm{C}_{2} \mathrm{~T}_{x}$ in $\mathrm{LP} 30$. Adapted, with permission, from $[17,50,51,57]$.

A recent breakthrough was made by Wang and colleagues [57], who investigated the influence of various solvents on the charge-storage mechanism in a macroporous $\mathrm{Ti}_{3} \mathrm{C}_{2} \mathrm{MXene}$ electrode, using the same $1 \mathrm{M} \mathrm{Li-TFSI} \mathrm{salt} \mathrm{concentration.} \mathrm{As} \mathrm{shown} \mathrm{in} \mathrm{Figure} \mathrm{4C,} \mathrm{different} \mathrm{CV} \mathrm{signatures}$ were obtained depending on the solvent (DMSO, ACN, and PC) used; surprisingly, the best electrochemical performance was obtained in PC-based electrolyte, with a capacity of $130 \mathrm{mAh} \mathrm{g}^{-1}$ at $2 \mathrm{mV} \mathrm{s}^{-1}$ within a $2.4 \mathrm{~V}$ voltage window. In situ XRD coupled with molecular dynamics simulations suggested that desolvated Li ions were intercalated between the MXene layers in PC-based electroIytes, leading to excellent electrochemical performance, $80 \mathrm{mAh} \mathrm{g}^{-1}$ being still delivered at charge/ discharge rates of $1000 \mathrm{C}$. However, the co-intercalation of solvent molecules was evidenced together with $\mathrm{Li}^{+}$ions in DMSO and ACN systems due to the strong interactions between DMSO/ ACN solvent molecules and $\mathrm{Li}^{+}$ions, which accounts for the limited electrochemical performance in these electrolytes. This study provides a rationale route by considering solvents as an effective strategy to improve the pseudocapacitive performance of MXene electrodes as well as other electrodes.

Finally, the latest advances have been made by Li and colleagues, who prepared MXene materials from a molten salt route (called MS-MXenes) [17]. Using a molten $\mathrm{CuCl}_{2}$ Lewis acid as etching bath, they prepared MXene free of $-\mathrm{F}$ and $-\mathrm{OH}$ groups but with surface terminations only composed of $-\mathrm{Cl}$ and $-\mathrm{O}$. The control of the MXene surface groups led to a remarkable electrochemical performance in a Li-ion battery electrolyte. The mirror-like CV profiles shown in Figure 4D differ from the previously reported pseudocapacitive signatures with at least some hump-like redox 
peaks (Figure 4A-C). Instead, the charge storage mechanism in $\mathrm{MS}-\mathrm{Ti}_{3} \mathrm{C}_{2} \mathrm{~T}_{x} \mathrm{MXene}$ is achieved by an almost constant current during reduction and oxidation processes, similar to what is observed in a pseudocapacitive material, most of the capacity here being delivered below in the $0.2-2 \mathrm{~V}$ versus $\mathrm{Li}^{+} / \mathrm{Li}$ potential range. The discharge capacity reaches a value as high as $205 \mathrm{mAh} \mathrm{g}^{-1}$ at $0.5 \mathrm{mV} \mathrm{s}^{-1}$. Those remarkable performances make MXene materials now suitable to be used as negative electrodes in nonaqueous energy-storage devices.

\section{Concluding Remarks and Future Perspectives}

MXene chemistry is continuing to grow, with almost 30 compositions today and more being discovered routinely. Thanks to their key features (high accessible surface area and metallic-like electrical conductivity), 2D MXenes have emerged as promising candidates in many applications, one of the most promising being electrochemical energy storage. For instance, ultrahigh volumetric capacitance beyond $1500 \mathrm{~F} \mathrm{~cm}^{-3}$ can be achieved with $\mathrm{Ti}_{3} \mathrm{C}_{2}$ MXenes when used as EC electrodes in aqueous acidic electrolytes and high power can be obtained by designing specific 3D porous or vertically aligned MXene electrodes. However, the narrow potential window that can be reached in aqueous electrolytes limits the energy density of these devices and their further use in practical applications.

However, the fast, nondiffusion limited Li-ion intercalation observed with some metal oxides such as $\mathrm{MoO}_{3}$ or $\mathrm{Nb}_{2} \mathrm{O}_{5}$ has revitalized the interest for studying $\mathrm{MXenes}$ in lithium or sodium organic electrolytes. While the nature of the solvents plays an important role in the charge-storage mechanism, the surface composition of MXene also has a strong impact on their reactivity and electrochemical performance. Various synthesis methods have then been proposed to tune the MXene surface chemistry to increase the electrochemical performance of MXenes in nonaqueous electrolytes. The recent report of a new MXene synthesis route using Lewis molten salts as an etching solution has already led to unique electrochemical signature and performance for lithium intercalation. These performances are assumed to originate from the tuning of the surface terminations (mainly - $\mathrm{Cl}$, -O groups), as compared with the MXenes prepared by conventional methods containing $-\mathrm{F},-\mathrm{OH}$, and $\mathrm{O}$-rich groups. However, the road to success will need even more efforts in the following aspects (see Outstanding Questions):

(i) Taking the Lewis acid molten salt synthesis route as an example, environmentally friendly, safe, efficient, and scalable methods to broaden the MAX phases precursors and prepare MXenes with tunable surface compositions are needed.

(ii) About $70 \%$ of all MXene research work has focused on the first discovered MXene, which is $\mathrm{Ti}_{3} \mathrm{C}_{2} \mathrm{~T}_{x}$ [6]; other MXenes, although more difficult to prepare, must be investigated from both theoretical and experimental points of views.

(iii) Although MXene electrodes can achieve high capacitance in aqueous electrolytes, research efforts should be focused onto MXene in nonaqueous electrolytes considering the much wider potential window and recent promising results obtained. Fine-tuning the nature and content of MXene surface groups and controlling the interlayer spacing offer key opportunities for performance improvement.

(iv) To meet the simultaneous high-power and high-energy challenge of ECs and high-power batteries, designing 3D porous, vertically aligned, or other structured MXene electrodes to improve ionic transport paths in the electrode without scarifying too much the volumetric energy density is also an important research direction to develop in the few next years.
Outstanding Questions

How can we tune the synthesis process of MXene to have better control of the surface functional groups present on MXene surface?

Considering the strong influence of surface terminations on the electrochemical behavior of MXenes, how specifically do these functional groups affect the electrochemical process and performance?

In nonaqueous Li-ion battery electrolytes, what is the charge-storage mechanism at the origin of high rate performance (Li-desolvation)?

Despite the outstanding performance that has been achieved with $\mathrm{Ti}_{3} \mathrm{C}_{2} \mathrm{~T}_{x}$ MXene electrodes for electrochemical energy storage, and with the diversity of the MXene family, can other MXene materials deliver even better energy and power density?

\section{Acknowledgments}

Z.L. is supported by the Fundamental Research Funds for the Central Universities (YJ201886) and the National Natural Science Foundation of China (Grant No. 501902215). H.S. was supported by a grant from the China Scholarship Council (NO. 201608370077). P.S., P.L.T., and H.S. thank the Agence Nationale de la Recherche (Labex STORE-EX) for support. 


\section{References}

1. Anasori, B. et al. (2017) 2D metal carbides and nitrides (MXenes) for energy storage. Nat. Rev. Mater. 2, 16098

2. Verger, L. et al. (2019) MXenes: an introduction of their synthesis, select properties, and applications. Trends Chem. 1, 656-669

3. Sokol, M. et al. (2019) On the chemical diversity of the MAX phases. Trends Chem. 1, 210-223

4. Li, Y. et al. (2020) Multielemental single-atom-thick A layers in nanolaminated $\mathrm{V} 2(\mathrm{Sn}, \mathrm{A}) \mathrm{C}(\mathrm{A}=\mathrm{Fe}, \mathrm{Co}, \mathrm{Ni}, \mathrm{Mn})$ for tailoring magnetic properties. Proc. Natl. Acad. Sci. U. S. A. 117, 820-825

5. Li, M. and Huang, Q. (2020) Recent progress and prospects of ternary layered carbides/nitrides MAX phases and their derived two-dimensional nanolaminates MXenes. J. Inorg. Mater. 35 $1-7$

6. Gogotsi, Y. and Anasori, B. (2019) The rise of MXenes. ACS Nano 13, 8491-8494

7. Xiong, D. et al. (2018) Recent advances in layered $\mathrm{Ti}_{3} \mathrm{C}_{2} \mathrm{~T}_{x}$ MXene for electrochemical energy storage. Small 14, 1703419

8. Magnuson, M. and Mattesini, M. (2017) Chemical bonding and electronic-structure in MAX phases as viewed by X-ray spectroscopy and density functional theory. Thin Solid Films 621 108-130

9. Naguib, M. et al. (2011) Two-dimensional nanocrystals pro duced by exfoliation of $\mathrm{Ti}_{3} \mathrm{AlC}_{2}$. Adv. Mater. 23, 4248-4253

10. Ghidiu, M. et al. (2014) Conductive two-dimensional titanium carbide 'clay' with high volumetric capacitance. Nature 516, 78-81

11. Liu, F. et al. (2017) Preparation of $\mathrm{Ti}_{3} \mathrm{C}_{2}$ and $\mathrm{Ti}_{2} \mathrm{C}$ MXenes by fluoride salts etching and methane adsorptive properties. Appl. Surf. Sci. 416, 781-789

12. Halim, J. et al. (2014) Transparent conductive two-dimensiona titanium carbide epitaxial thin films. Chem Mat. 26, 2374-2381

13. Natu, $\mathrm{V}$. et al. (2020) $2 \mathrm{D} \mathrm{Ti}{ }_{3} \mathrm{C}_{2} \mathrm{~T}_{z} \mathrm{MXene}$ synthesized by water-free etching of $\mathrm{Ti}_{3} \mathrm{AlC}_{2}$ in polar organic solvents. Chem 6, 616-630

14. Li, T. et al. (2018) Fluorine-free synthesis of high-purity $\mathrm{Ti}_{3} \mathrm{C}_{2} \mathrm{~T}_{x}$ $(\mathrm{T}=\mathrm{OH}, \mathrm{O})$ via alkali treatment. Angew. Chem. Int. Ed. 57. 6115-6119

15. Pang, S.-Y. et al. (2019) Universal strategy for HF-free facile and rapid synthesis of two-dimensional MXenes as multifunctional energy materials. J. Am. Chem. Soc. 141, 9610-9616

16. Alhabeb, M. et al. (2018) Selective etching of silicon from $\mathrm{Ti}_{3} \mathrm{SiC}_{2}$ (MAX) to obtain 2D titanium carbide (MXene). Angew. Chem. 130, 5542-5546

17. Li, Y. et al. (2020) A general Lewis acidic etching route for preparing MXenes with enhanced electrochemical performance in non-aqueous electrolyte. Nat. Mater. Published online April 13 2020. https://doi.org/10.1038/s41563-020-0657-0

18. Persson, P.O.A. and Rosen, J. (2019) Current state of the art on tailoring the MXene composition, structure, and surface chemistry. Curr. Opin. Solid State Mater. Sci. 23, 100774

19. Hope, M.A. et al. (2016) NMR reveals the surface functionalisation of $\mathrm{Ti}_{3} \mathrm{C}_{2}$ MXene. Phys Chem Phys. 18, 5099-5102

20. Hu, M. et al. (2018) Surface functional groups and interlaye water determine the electrochemical capacitance of $\mathrm{Ti}_{3} \mathrm{C}_{2} \mathrm{~T}$ MXene. ACS Nano 12, 3578-3586

21. Hart, J.L. et al. (2019) Control of MXenes' electronic properties through termination and intercalation. Nat. Commun. 10, 522

22. Zhang, K. et al. (2020) Enhancing the magnetism of 2D carbide MXene $\mathrm{Ti}_{3} \mathrm{C}_{2} \mathrm{~T}_{x}$ by $\mathrm{H}_{2}$ annealing. Carbon 157, 90-96

23. Lee, Y. et al. (2020) Oxidation-resistant titanium carbide MXene films. J. Mater. Chem. A 8, 573-581

24. Persson, I. et al. (2020) How much oxygen can a MXene surface take before it breaks? Adv. Funct. Mater. Published online February 18, 2020. https://doi.org/10.1002/adfm.201909005

25. Khazaei, M. et al. (2017) Electronic properties and applications of MXenes: a theoretical review. J. Mater. Chem. C 5, 2488-2503

26. Fan, Z. et al. (2018) A nanoporous MXene film enables flexible supercapacitors with high energy storage. Nanoscale 10 , 9642-9652

27. Zhang, C. et al. (2017) Transparent, flexible, and conductive 2D titanium carbide (MXene) films with high volumetric capacitance. Adv. Mater. 29, 1702678

28. Lukatskaya, M.R. et al. (2013) Cation intercalation and high volumetric capacitance of two-dimensional titanium carbide. Science 341, 1502-1505
29. Lukatskaya, M.R. et al. (2017) Ultra-high-rate pseudocapacitive energy storage in two-dimensional transition metal carbides. Nat. Energy 2, 17105

30. Lukatskaya, M.R. et al. (2015) Probing the mechanism of high capacitance in $2 \mathrm{D}$ titanium carbide using in situ X-ray absorption spectroscopy. Adv. Energy Mater. 5, 1500589

31. Hu, M. et al. (2016) High-capacitance mechanism for $\mathrm{Ti}_{3} \mathrm{C}_{2} \mathrm{~T}_{x}$ MXene by in situ electrochemical Raman spectroscopy investigation. ACS Nano 10, 11344-11350

32. Xia, Y. et al. (2018) Thickness-independent capacitance of vertically aligned liquid-crystalline MXenes. Nature 557, 409-412

33. VahidMohammadi, A. et al. (2019) Assembling 2D MXenes into highly stable pseudocapacitive electrodes with high power and energy densities. Adv. Mater. 31, 1806931

34. Shan, Q. et al. (2018) Two-dimensional vanadium carbide $\left(V_{2} C\right)$ MXene as electrode for supercapacitors with aqueous electrolytes. Electrochem. Commun. 96, 103-107

35. Rakhi, R.B. et al. (2015) Effect of postetch annealing gas composition on the structural and electrochemical properties of $\mathrm{Ti}_{2} \mathrm{CT}_{x}$ MXene electrodes for supercapacitor applications. Chem. Mat. $27,5314-5323$

36. Halim, J. et al. (2016) Synthesis and characterization of 2D molybdenum carbide (MXene). Adv. Funct. Mater. 26, 3118-3127

37. Tao, Q. et al. (2017) Two-dimensional $\mathrm{Mo}_{13} \mathrm{C}$ MXene with divacancy ordering prepared from parent 3D laminate with inplane chemical ordering. Nat. Commun. 8, 14949

38. Zheng, S. et al. (2019) lonic liquid pre-intercalated MXene films for ionogel-based flexible micro-supercapacitors with high volumetric energy density. J. Mater. Chem. A 7, 9478-9485

39. Lin, Z. et al. (2016) Capacitance of $\mathrm{Ti}_{3} \mathrm{C}_{2} \mathrm{~T}_{x} \mathrm{MXene}$ in ionic liquid electrolyte. J. Power Sources 326, 575-579

40. Dall'Agnese, Y. et al. (2016) Capacitance of two-dimensional titanium carbide (MXene) and MXene/carbon nanotube composites in organic electrolytes. J. Power Sources 306, 510-515

41. Lin, Z. et al. (2016) Electrochemical and in-situ X-ray diffraction studies of $\mathrm{Ti}_{3} \mathrm{C}_{2} \mathrm{~T}_{X} \mathrm{MXene}$ in ionic liquid electrolyte. Electrochem. Commun. 72, 50-53

42. Lin, Z. and Simon, P. (2019) MXenes for Supercapacitor Application In 2D Metal Carbides and Nitrides (MXenes): Structure, Properties and Applications (Babak, A. and Yury, G., eds), pp. 349-365, Springer

43. Xu, K. et al. (2018) Tracking ionic rearrangements and interpreting dynamic volumetric changes in two-dimensiona metal carbide supercapacitors: a molecular dynamics simulation study. Chem SusChem 11, 1892-1899

44. Jäckel, N. et al. (2016) Electrochemical in situ tracking of volumetric changes in two-dimensional metal carbides (MXenes) in ionic liquids. ACS Appl. Mater. Interfaces 8, 32089-32093

45. Augustyn, V. et al. (2013) High-rate electrochemical energy storage through $\mathrm{Li}^{+}$intercalation pseudocapacitance. Nat. Mater. $12,518-522$

46. Brezesinski, T. et al. (2010) Ordered mesoporous $\mathrm{a}-\mathrm{MoO}_{3}$ with iso-oriented nanocrystalline walls for thin-film pseudocapacitors. Nat. Mater. 9, 146-151

47. Naguib, M. et al. (2012) MXene: a promising transition metal carbide anode for lithium-ion batteries. Electrochem. Commun. 16, 61-64

48. Come, J. et al. (2012) A non-aqueous asymmetric cell with a Ti2 based two-dimensional negative electrode. J. Electrochem. Soc. 159, A1368-A1373

49. Mashtalir, O. et al. (2013) Intercalation and delamination of layered carbides and carbonitrides. Nat. Commun. 4, 1716

50. Cheng, R. et al. (2019) Understanding the lithium storage mechanism of $\mathrm{Ti}_{3} \mathrm{C}_{2} \mathrm{~T}_{x}$ MXene. J. Phys. Chem. C 123, 1099-1109

51. Dall'Agnese, Y. et al. (2015) Two-dimensional vanadium carbide (MXene) as positive electrode for sodium-ion capacitors. J. Phys. Chem. Lett. 6, 2305-2309

52. Wang, X. et al. (2015) Pseudocapacitance of MXene nanosheets for high-power sodium-ion hybrid capacitors. Nat. Commun. 6, 6544

53. Kajiyama, S. et al. (2016) Sodium-ion intercalation mechanism in MXene nanosheets. ACS Nano 10, 3334-3341

54. Xie, X. et al. (2016) Porous heterostructured MXene/carbon nanotube composite paper with high volumetric capacity for sodium-based energy storage devices. Nano Energy 26 513-523 
55. Ming, F. et al. (2019) Porous MXenes enable high performance potassium ion capacitors. Nano Energy 62, 853-860

56. Xie, X. et al. (2018) Porous $\mathrm{Ti}_{3} \mathrm{C}_{2} \mathrm{~T}_{X} \mathrm{MXene}$ for ultrahigh-rate sodium-ion storage with long cycle life. ACS Appl. Nano Mater. $1,505-511$

57. Wang, X. et al. (2019) Influences from solvents on charge storage in titanium carbide MXenes. Nat. Energy 4, 241-248

58. Mathis, T.S. et al. (2019) Energy storage data reporting in perspective - guidelines for interpreting the performance of electrochemical energy storage systems. Adv. Energy Mater. 9 1902007
59. Mitchell, J.B. et al. (2019) Confined interlayer water promotes structural stability for high-rate electrochemical proton intercalation in tungsten oxide hydrates. ACS Energy Lett. 4, 2805-2812

60. Ardizzone, S. et al. (1990) "Inner" and "outer" active surface of $\mathrm{RuO}_{2}$ electrodes. Electrochim. Acta 35, 263-267

61. Shao, H. et al. (2019) Electrochemical study of pseudocapacitive behavior of $\mathrm{Ti}_{3} \mathrm{C}_{2} \mathrm{~T}_{x} \mathrm{MX}$ Xene material in aqueous electrolytes. Energy Stor. Mater. 18, 456-461

62. Wang, J. et al. (2007) Pseudocapacitive contributions to electrochemical energy storage in $\mathrm{TiO}_{2}$ (anatase) nanoparticles. J. Phys. Chem. C 111, 14925-14931 